\title{
Landslide Area Detection from Synthetic Aperture Radar Images Using Convolutional Adversarial Autoencoder and One-class SVM
}

\author{
Shingo Mabu \\ Graduate School of Sciences and Technology for Innovation, Yamaguchi University \\ 2-16-1 Tokiwadai, Ube, Yamaguchi 755-8611, Japan \\ Soichiro Hirata \\ Graduate School of Sciences and Technology for Innovation, Yamaguchi University \\ 2-16-1 Tokiwadai, Ube, Yamaguchi 755-8611, Japan \\ Takashi Kuremoto \\ Graduate School of Sciences and Technology for Innovation, Yamaguchi University \\ 2-16-1 Tokiwadai, Ube, Yamaguchi 755-8611, Japan \\ E-mail:mabu@yamaguchi-u.ac.jp,wu@yamaguchi-u.ac.jp
}

\begin{abstract}
An anomaly detection model using deep learning for detecting disaster-stricken (landslide) areas in synthetic aperture radar images is proposed. Since it is difficult to obtain a large number of training images, especially disaster area images, with annotations, we design an anomaly detection model that only uses normal area images for the training, where the proposed model combines a convolutional adversarial autoencoder and one-class SVM. In the experiments, the ability in detecting normal and abnormal areas is evaluated.
\end{abstract}

Keywords: anomaly detection, convolutional neural network, adversarial autoencoder, one-class SVM, synthetic aperture radar, landslide

\section{Introduction}

In Japan, typhoons often approach and pass from July to September every year because of the effects of westerly winds and high atmospheric pressures. Landslide disasters frequently occur due to heavy rains caused by typhoons, which leads to major accidents related to transportation and human life. Until now, observation of the disaster areas, e.g., landslides, has been mainly conducted by aircraft ${ }^{1}$. However, in recent years, remote sensing using satellite images has attracted attention as a method for observing a wide $\operatorname{area}^{2}$. In satellite remote sensing, a sensor is mounted on an artificial satellite to observe the ground surface of the earth, and the obtained image is analyzed. Therefore, we can see the damages without going directly to the disaster areas. Typical satellite images include optical images that are obtained from sunlight reflection, and synthetic aperture radar (SAR) images that are obtained by sensors that emit microwaves to the ground surface of the earth. It is easy for a human to interpret optical images, but they cannot be observed at night or in bad weather. On the other hand, SAR can observe the surface of the earth regardless of time and weather; thus, SAR images are useful for rapid rescue activities at night and in bad weather conditions. However, it is difficult for human eyes to interpret SAR images, unlike optical images. Therefore, many methods that analyze SAR images have been proposed to detect disaster areas rapidly ${ }^{3,4}$, and machine learning techniques, especially deep learning, have also been applied to landslide area detection ${ }^{5}$.

When deep learning is applied to disaster area detection, a large number of training samples are necessary. However, shooting conditions of SAR images are (C) The 2021 International Conference on Artificial Life and Robotics (ICAROB2021), January 21 to 24, 2021 
different time by time, e.g., seasons, and it is also difficult to obtain a sufficient number of training samples with annotation (correct class labels) immediately after a disaster occurs. To overcome this problem, we propose an anomaly detection system that detects abnormal images that deviate from the features of normal images. The proposed method uses only normal images for the training without using abnormal (disaster) images. Normal images are relatively easier to obtain compared with abnormal images, thus anomaly detection is useful in a practical situation of disaster area detection. The proposed method consists of a feature extraction part and a detection part. The feature extraction is realized by convolutional adversarial autoencoder (CAAE) that is the extension of adversarial autoencoder $(\mathrm{AAE})^{6}$, where the layers of AAE are replaced with convolution and deconvolution layers. CAAE is trained with unsupervised learning, thus the cost of the annotation is not necessary. The detection part is realized by one-class SVM (OCSVM $)^{7}$ that detects outliers that deviate from the normal region.

This paper is organized as follows. In section 2, the mechanism and features of SAR is introduced. In Section 3 , the proposed method that combines CAAE and OCSVM is explained. In section 4, the experimental conditions and results are described. Finally, section 5 is devoted to conclusions.

\section{Synthetic Aperture Radar (SAR)}

SAR is an active image radar that synthesizes small antennas mounted on a platform, such as an aircraft or satellite, to realize large virtual antennas and generates high-resolution radar images ${ }^{8,9,10)}$. Because SAR is an active sensor that emits microwaves, it is possible to observe the surface of the earth regardless of the presence or absence of sunlight and clouds. SAR images are applied to the research fields of agriculture, disaster, oceans, earth science, and so on ${ }^{11,12)}$. SAR emits microwaves and receives the reflected microwaves from the surface of the earth (Fig. 1). When a microwave emitted from the SAR antenna enters a conductor or dielectric, a current is induced, and the microwave is reemitted from the induced current. This is called scattering and scattering in the opposite direction of the incident wave is called backscattering. Because backscattering is the diffuse reflection caused by scattering, backscattering is different from specular reflection.

SAR receives the backscattering and executes image reproduction. The scattering intensity of the microwaves strongly depends on the frequency, wavelength and

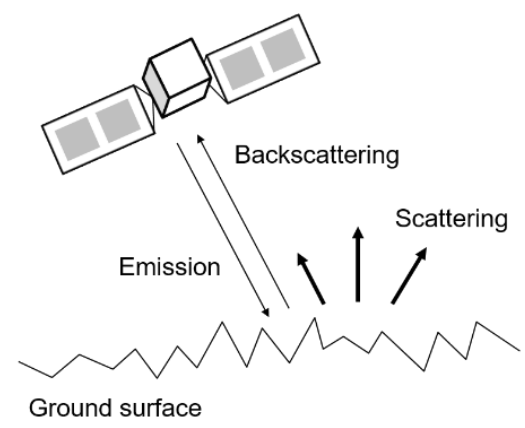

Fig. 1. Microwave emission, Scattering, and backscattering of SAR $^{10}$

electric characteristics (dielectric constant, etc.) of the scatterer. Therefore, for example, seawater or cars made of metal strongly reflect microwaves because a current is easily induced. On the other hand, sand and trees have low reflectivity because hardly any current is induced. In each pixel of SAR images, the intensity of the received microwave is recorded.

\section{Materials and methods}

\subsection{Dataset}

A SAR image used in this paper is shown in Fig. 2. It shows the northern Kyushu area in Japan on July 7, 2017, taken by Advanced Land Observing Satellite No. 2 (ALOS-2). Large-scale landslides due to the torrential rain from July 5 to 6,2017 , occurred in this area. The yellow areas in Fig. 2 show the locations of the landslide that were annotated by the Geospatial Information Authority of Japan. The size of the image is $6648 \times$ 4360 [pixels] and the resolution per pixel is about $3 \mathrm{~m}^{2}$. In this paper, ROI (region of interest)-based anomaly detection is carried out, that is, we first divided the whole

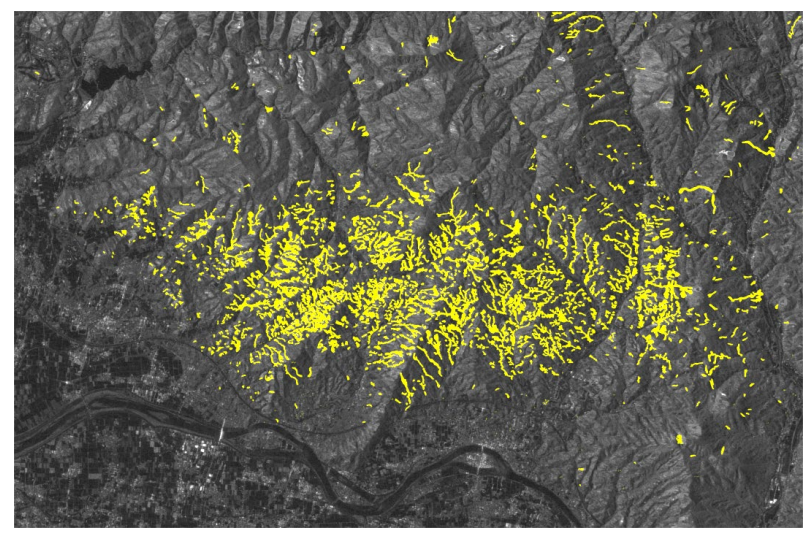

Fig. 2. SAR image and landslide areas (yellow areas) 


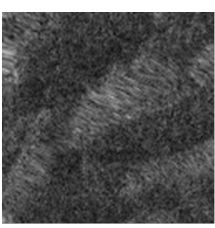

Normal

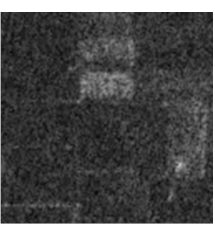

Abnormal
Fig. 3. Examples of $128 \times 128$ [pixels] ROI images

SAR image into $128 \times 128$ [pixels] ROI images. Fig. 3 shows examples of normal and abnormal ROI images. Note that the target area for the detection of normal and abnormal is the central $32 \times 32$ [pixels] area of each ROI image. In other words, the surrounding area of the target area is used as a piece of supporting information (context) for the detection. The ROI images were split into training data including normal only, testing data of normal, and testing data of abnormal.

\subsection{Method}

The overview of the anomaly detection model designed in this paper is shown in Fig. 4, where CAAE generates feature fector $\boldsymbol{z}, \boldsymbol{z}$ is transformed by principal component analysis (PCA), and OCSVM classifies $\boldsymbol{Z}$ as normal or abnormal.

\subsubsection{Feature extraction using Convolutional Adversarial Autoencoder (CAAE)}

In this paper, the extended model of $\mathrm{AAE}^{6}$, named CAAE, is used for the feature extraction of SAR images. Fig. 5

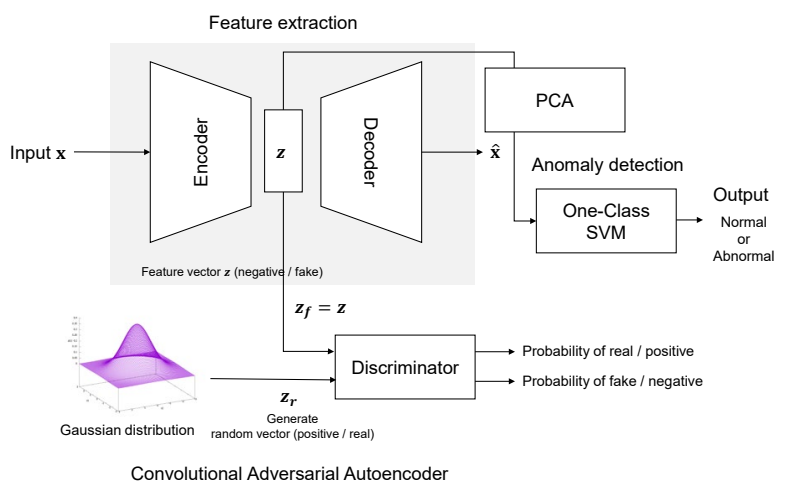

Fig. 4. Examples of $128 \times 128$ [pixels] ROI images

shows the detailed structure of CAAE that extracts features from ROI images. The upper part of Fig. 5 is a convolutional autoencoder (CAE) that contains an encoder and a decoder. The encoder outputs a 128dimensional feature vector $\boldsymbol{z}$, and the decoder outputs the reconstructed ROI images. The encoder consists of some Block1 and fully-connected layers, where a Block1 contains convolution and max pooling layers along with batch normalization and rectified linear unit (ReLU). The decoder consists of some Block2, fully-connected and deconvolution layers, where a Block2 contains a deconvolution layer along with batch normalization and ReLU. The loss function (LOSS1 in Fig. 5) is the mean squared error between the input and output images, and the weights of the encoder and decoder are updated by error backpropagation with Adam ${ }^{13}$ to minimize LOSS1. The encoder and discriminator at the lower part of Fig. 5 are adversarial networks, where the discriminator

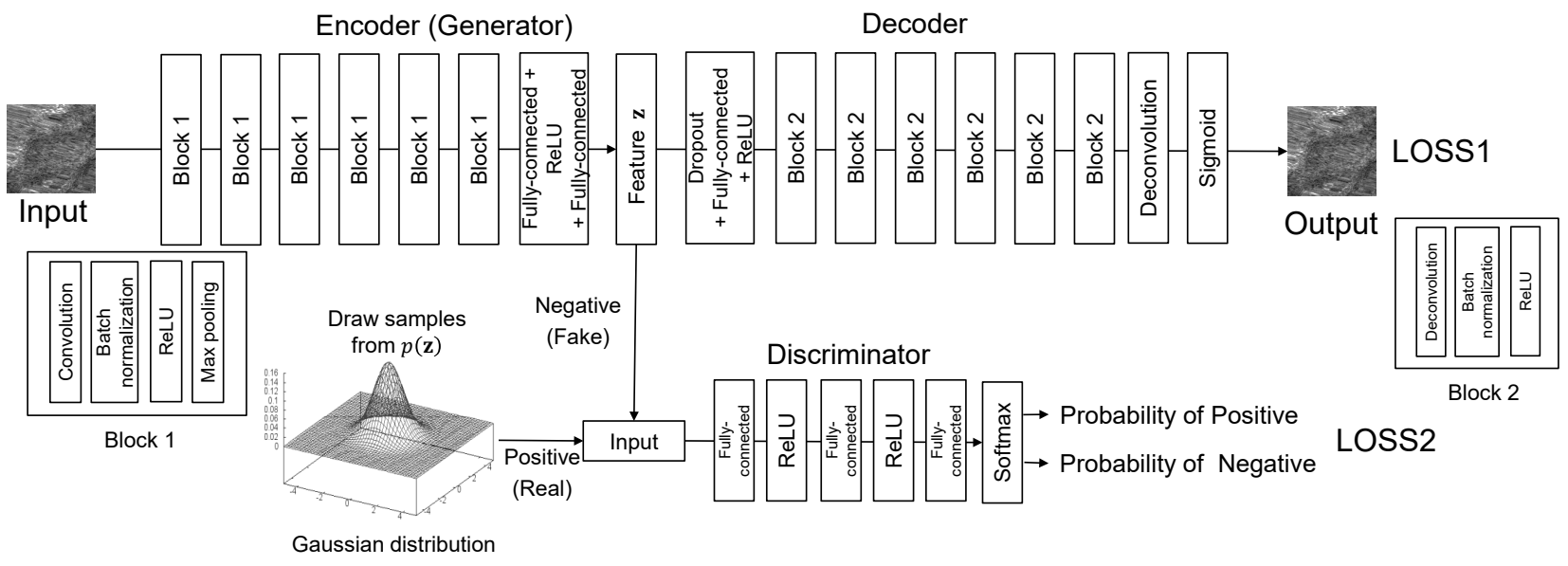

Fig. 5. Detailed structure of CAAE 

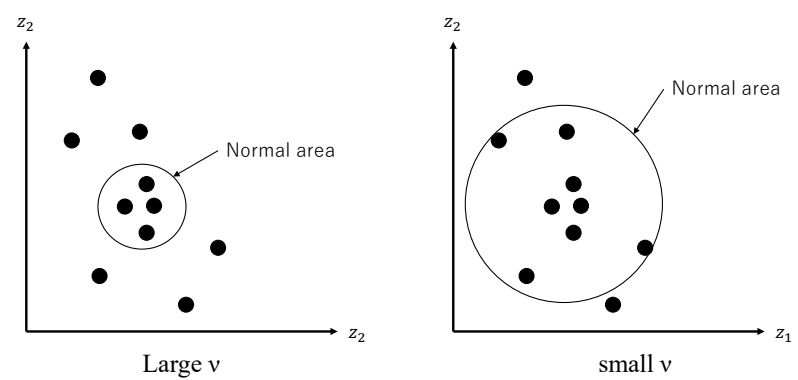

Fig. 6. Effects of $v$ in OCSVM

distinguishes the vectors generated by Gaussian distribution (regarded as positive or real samples) from the feature vector $\boldsymbol{z}$ inputted from the encoder (regarded as negative or fake samples). The loss function (LOSS2 in Fig. 5) is the softmax cross-entropy based on the positive and negative probabilities outputted by the discriminator. The discriminator updates the weights to minimize LOSS2, while the encoder updates the weights to maximize LOSS2. As a result of the above learning, the feature vector $\boldsymbol{z}$ captures the features of the input images and its distribution follows a Gaussian distribution. The constraint imposed by the adversarial networks increases the robustness of the feature extraction executed by the convolutional autoencoder.

\subsection{Anomaly detection by One-class SVM (OCSVM)}

The feature vector $\boldsymbol{z}$ generated by CAAE is inputted to OCSVM. SVM is originally a supervised-learning-based classification algorithm for two classes, which needs training data with class labels. OCSVM needs only oneclass data for the training, and after the training, OCSVM regards the data that deviates from the learned class data as outliers, that is, abnormal. Anomaly detection for the testing images in this paper is carried out by OCSVM. OCSVM has a parameter $v$ that determines the proportion of normal and abnormal areas. For example, when $v$ is set at a large value as shown in Fig. 6 (left), the normal area becomes small. In this case, only the data that is very near to the center of the normal area is regarded as normal, which increases the sensitivity of abnormal, but may increase false positives, that is, the true normal (negative) is misclassified as abnormal (positive). Conversely, when $v$ is small, the normal area becomes large, which increases the specificity, but may increase false negatives, that is, the true abnormal is regarded as normal. Therefore, the value of $v$ should be determined carefully considering the trade-off between false positive and false negative.
Table 1. The number of ROI images in condition 1

\begin{tabular}{lr}
\hline Training/Testing & The number of images \\
\hline Training (normal only) & 13542 \\
Testing (normal) & 164 \\
Testing (abnormal) & 164 \\
\hline
\end{tabular}

\section{Experiments}

\subsection{Condition 1}

The class labels of ROIs are given by the following rule. When $60 \%$ or more area of the central $32 \times 32$ area of each ROI image contains the abnormal area, it is regarded as abnormal, otherwise, normal. The number of training data (normal only), that of testing data (normal), and that of testing data (abnormal) are shown in Table 1. CAAE is trained with the training data and all the training data are encoded as the feature vectors. Then, PCA is applied to the feature vectors to reduce the number of dimensions from 128 to 40 . The feature vectors after the dimensional reduction by PCA are used to train OCSVM. Finally, the testing images encoded and transformed by CAAE and PCA are classified as normal and abnormal.

Table 2 shows the results obtained by the combination of CAE, PCA, and OCSVM (called conventional method), and Table 3 shows those obtained by the combination of CAAE, PCA, and OCSVM (called proposed method). The best accuracy obtained by the conventional method is $55.7 \%(v=0.5)$ and that obtained by the proposed method is $57.9 \%(v=0.4)$. Therefore, we can confirm that CAAE is better than CAE as a feature extraction method, however, there is a large room for improvement on the accuracy.

\subsection{Condition 2}

In condition 2, the class labels of ROIs are given by the following rule. The labeling rule of abnormal areas is the same as condition 1 , that is, when $60 \%$ or more area of each ROI image contains abnormal, it is regarded as abnormal. In the case of the labeling of normal, ROIs without containing any abnormal areas are regarded as normal. In condition 1 , the threshold that separates the normal and abnormal ROIs is $60 \%$, which means that even if the ROIs contains $59 \%$ abnormal areas, they should be classified as normal. To clearly separate the 
Table 2. Results obtained by CAE + PCA + OCSVM (Conventional method) in condition 1

\begin{tabular}{|c|c|c|c|c|c|c|c|}
\hline & & \multicolumn{6}{|c|}{$v$} \\
\hline & & 0.2 & 0.3 & 0.4 & 0.5 & 0.6 & 0.7 \\
\hline \multirow{3}{*}{ 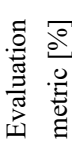 } & Accuracy & 53.4 & 54.9 & 55.6 & 55.7 & 49.4 & 47.4 \\
\hline & Sensitivity & 23.4 & 32.1 & 41.1 & 51.1 & 56.1 & 63.8 \\
\hline & Specificity & 83.4 & 77.8 & 70.1 & 60.3 & 42.7 & 31.1 \\
\hline
\end{tabular}

Table 3. Results obtained by CAAE + PCA + OCSVM (Proposed method) in condition 1

\begin{tabular}{|c|c|c|c|c|c|c|c|c|}
\hline & & & \multicolumn{6}{|c|}{$v$} \\
\hline & & & 0.2 & 0.3 & 0.4 & 0.5 & 0.6 & 0.7 \\
\hline \multirow{3}{*}{ 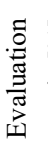 } & $\sigma^{\circ}$ & Accuracy & 55.9 & 56.6 & 57.9 & 57.4 & 54.4 & 52.4 \\
\hline & $\mathscr{E}$ & Sensitivity & 24.5 & 31.5 & 37.1 & 39.4 & 41.9 & 47.2 \\
\hline & $\Xi$ & Specificity & 87.3 & 81.6 & 78.7 & 75.4 & 66.9 & 57.6 \\
\hline
\end{tabular}

features of normal and abnormal, condition 2 adopts the above labeling rule. Table 4 shows the numbers of training and testing data. The procedure of executing CAAE, PCA and OCSVM is the same as condition 1 .

Table 5 shows the results of the conventional method and Table 6 shows those of the proposed method. The best accuracy obtained by the conventional method is $61.4 \%$ $(v=0.2)$ and that obtained by the proposed method is $66.0 \%(v=0.5)$; thus we can see that the accuracy becomes better than condition 1 and the accuracy obtained by CAAE is better than that by CAE.

\section{Conclusions}

In this paper, we proposed an anomaly detection method for classifying normal (non-disaster) areas and abnormal (disaster/landslide) areas, where the proposed method consists of CAAE, PCA, and OCSVM. In the experiments, we evaluated the detection performance in the two conditions. From the results, it was clarified that CAAE is better than CAE as a feature extraction method. The remaining problem is the classification of ROI images that contain small abnormal areas. Since it is difficult to make a sharp boundary between normal and
Table 4. The number of ROI images in condition 2

\begin{tabular}{lr}
\hline Training/Testing & The number of images \\
\hline Training (normal only) & 7967 \\
Testing (normal) & 164 \\
Testing (abnormal) & 164 \\
\hline
\end{tabular}

Table 5. Result obtained by CAE + PCA + OCSVM (Conventional method) in condition 2

\begin{tabular}{|c|c|c|c|c|c|c|c|}
\hline & & \multicolumn{6}{|c|}{$v$} \\
\hline & & 0.2 & 0.3 & 0.4 & 0.5 & 0.6 & 0.7 \\
\hline \multirow{3}{*}{ 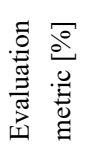 } & Accuracy & 61.4 & 60.4 & 61.2 & 59.8 & 58.7 & 56.2 \\
\hline & Sensitivity & 39.5 & 47.7 & 56.6 & 64.3 & 68.1 & 73.3 \\
\hline & Specificity & 83.3 & 73.1 & 65.8 & 55.5 & 49.3 & 39.1 \\
\hline
\end{tabular}

Table 6. Result obtained by CAAE + PCA + OCSVM (Proposed method) in condition 2

\begin{tabular}{|c|c|c|c|c|c|c|c|}
\hline & & \multicolumn{6}{|c|}{$v$} \\
\hline & & 0.2 & 0.3 & 0.4 & 0.5 & 0.6 & 0.7 \\
\hline \multirow{3}{*}{ 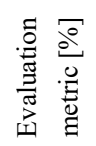 } & Accuracy & 64.0 & 64.8 & 65.5 & 66.0 & 62.7 & 59.6 \\
\hline & Sensitivity & 39.6 & 44.4 & 50.0 & 54.8 & 56.9 & 60.6 \\
\hline & Specificity & 88.3 & 85.1 & 80.9 & 77.2 & 68.5 & 58.5 \\
\hline
\end{tabular}

abnormal, other method such as segmentaion models would be useful. In addition, the proposed method should be evaluated on other areas of disasters to confirm the generalization ability.

\section{Acknowledgements}

This work was supported by JSPS KAKENHI Grant Number JP20H02417.

\section{References}

1. T. Lillesand, R. W. Kiefer, J. Chipman, Remote sensing and image interpretation, John Wiley \& Sons, 2014 
2. Y, Liu and $\mathrm{L}$. Wu, Geological disaster recognition on optical remote sensing images using deep learning, Procedia Computer Science 91, 2016, pp. 566-575

3. H. Shahabi, M. Hashim, Landslide susceptibility mapping using gis-based statistical models and remote sensing data in tropical environment, Scientific reports 5, 9899, 2015

4. N. Casagli, V. Tofani, A. Ciampalini, F. Raspini, P. Lu, S. Morelli, Txt-tool 2.039-3.1: Satellite remote sensing techniques for landslides detection and mapping, Landslide Dynamics: ISDR-ICL Landslide Interactive Teaching Tools, Springer, 2018, pp. 235-254

5. P. Liu, Y. Wei, Q. Wang, Y. Chen, J. Xie, Research on post-earthquake landslide extraction algorithm based on improved u-net model, Remote Sensing 12(5), 894, 2020

6. A. Makhzani, J. Shlens, N. Jaitly and I. J. Goodfellow, Adversarial Autoencoders, International Conference on Learning Representations, 2016

7. L. M. Manevitz and M. Yousef, One-Class SVMs for Document Classification, Journal of Machine Learning Research, 2001, pp. 139-154

8. J. P. Fitch, Synthetic aperture radar, Springer Science \& Business Media, 2012

9. K. Ouchi, Principles of synthetic aperture radar for remote sensing, Tokyo Denki University Press, 2009 (in Japanese)

10. S. Mabu, Y. Nakayama and T. Kuremoto, Landslide Classification from Synthetic Aperture Radar Images Using Convolutional Neural Network with Multichannel Information, Journal of Signal Processing, 24(2), 2020, pp. 61-73

11. C. Atzberger, Advances in remote sensing of agriculture: Context description, existing operational monitoring systems and major information needs, Remote Sensing, 5(2), 2013, pp.949-981

12. E. C. Barret, Introduction to environmental remote sensing, Routledge, 2013

13. D. Kingma and J. Ba, Adam: A method for stochastic optimization. arXiv preprint arXiv:1412.6980, 2014 